\author{
A Balance Theory Approach to Group Problem Solving \\ Gbemisola A. Abimbola, P. Robert Duimering*, Zhehui Zhong \\ University of Waterloo
}

Under review

November 2006

\begin{abstract}
* Corresponding author: P. Robert Duimering, Assistant Professor, Department of Management Sciences, University of Waterloo, Waterloo, Ontario, N2L 3G1, Canada. Telephone: (519) 8884567 ext. 32831. Fax: (519) 746-7252. Email: rduimering@uwaterloo.ca.

This research was funded by Bell University Labs at the University of Waterloo. We thank Frank Safayeni for introducing us to Alex Bavelas' five squares problem, which inspired the method developed here. This paper is based on research conducted as part of Gbemisola Abimbola's MASc thesis at the University of Waterloo.
\end{abstract}




\title{
A Balance Theory Approach to Group Problem Solving
}

\begin{abstract}
This paper presents a view of group problem solving based on Heider's balance theory (Cartwright \& Harary, 1956; Doreian, Kapuscinski, Krackhardt, \& Szczypula, 1996; Heider, 1946, 1958), which conceptualizes the problem solving process as a progression towards increasing structural balance. Balance theory provides a means of representing problems in ways that capture both gestalt restructuring and incremental search properties of problem solving. Results of a group problem solving experiment are reported, which investigates the effects of certain properties of problem structure on problem solving behavior and performance.
\end{abstract}

\section{Introduction}

Group problem solving is pervasive in organizations and social policy settings, playing a role in decision making, strategy formulation, the development of new products, services or policies, and adaptation to changing conditions in institutional environments. However, very little is known about either the behavioral processes involved or the factors that affect performance. Recent reviews of the literature on group behavior indicate virtually no empirical studies of group problem solving (Cohen \& Bailey, 1997; Ilgen, Hollenbeck, Johnson, \& Jundt, 2005). Laboratory studies have examined the effect of group size and problem abstractness on group problem solving performance (Hendrick, 1979; Holloman \& Hendrick, 1971) and shown that groups can outperform individuals on the same problems under certain conditions (Laughlin, Zander, Knievel, \& Tan, 2003; Laughlin, Hatch, Silver \& Boh, 2006). The latter results have been used to justify group rather than individual problem solving strategies in organizations, but 
their generalizability depends on the characteristics of the problem being solved. For example, a prominent feature of group problems in organizations is the distribution of information among group members, so communication and the sharing and integration of distributed information are required to reach a solution. Experiments comparing individual and group performance, however, tend to use problem stimuli for which all individuals have access to complete information. Thus, communication is unproblematic in such studies, though it is a central aspect of empirical group problem situations. A series of classic experiments originated by Bavelas (1950) and Leavitt (1951) used groups of five participants connected in line, wheel, circle, etc., arrangements to investigate the effects of communication network structures on group task performance. However, to isolate the effects of communication structure these studies typically involved relatively simple information processing tasks as stimuli rather than conceptually challenging group problems (Bavelas, 1973; Bavelas, Hastorf, Gross, \& Kite, 1965).

This paper has three goals: 1) to introduce a conceptual view of group problem solving based on Heider's balance theory (Cartwright \& Harary, 1956; Heider, 1946, 1958) which conceptualizes problem solving as a progression towards increasing structural balance (or cognitive consistency; Abelson et al., 1968), 2) to describe an experimental method and stimulus for studying group problem solving based on this conceptual framework, 3) to report the results of an experiment investigating the effects of certain properties of problem structure on group problem solving behavior and performance.

\section{Background}

\section{Problem solving}

Problems and routine tasks are both goal-directed human activities, but they differ in the degree to which the person or group performing the activity understands the steps (or means- 
ends relations) required to move from the initial state to the desired goal state (Dunbar, 1988; Swinth \& Tuggle, 1971). Routine tasks are structured activities in which the path taken to achieve the goal is essentially known; problems are unstructured or incorrectly structured activities in which the appropriate path is unknown, ambiguous or incorrectly understood. The problem solving literature has been dominated by two theoretical approaches which emphasize distinct aspects of the process and differ in their treatment of the idea of problem structure. Gestalt psychologists (Kohler, 1969; Wertheimer, 1982/45) emphasized the role of insight associated with a sudden restructuring of the cognitive field during problem solving (and corresponding "ah hah!" experience), typified by the solution of the famous "nine-dot problem" (MacGregor, Ormerod, \& Chronicle, 2001). Essentially they argued that the fundamental psychological process involved in problem solving was the recognition or formulation of an appropriate problem structure enabling the solution to be obtained. Experiments based on this view generally use so-called "insight problems" as stimuli, which can be solved in a single step such that recognizing the appropriate problem structure coincides with obtaining the solution. The information processing systems approach of Newell and Simon (1972) emphasized the role of incremental search within a problem space, typified by the solution to the "towers of Hanoi" problem. This approach assumes that the basic dimensions of the problem structure are understood a priori, so the problem solving process amounts to exploring the associated problem space until the solution is found. Problem solving difficulty increases with the size of the search space, so this approach has been applied most successfully in rule-based artificial intelligence applications where large-scale but well-defined problems (e.g., chess playing) can be solved by computers using trial and error search. 
The problem solving literature has two basic limitations that this paper attempts to address. First, although it is generally accepted that both gestalt restructuring and incremental search are involved in problem solving, researchers have had limited success integrating these two approaches (Kaplan \& Simon, 1990; MacGregor et al., 2001; Metcalfe \& Wiebe, 1987). No suitable theory has been proposed that places both aspects of the process in a common conceptual framework. Methodological limitations also likely account for this lack of integration, since experiments tend to be designed to investigate either insight or incremental search but not both (e.g., it is difficult to study search in insight problems since problem structure and solution are obtained almost simultaneously). In addition, few methods have been developed that enable the direct experimental manipulation of problem structure as an independent variable. Furthermore, observational limitations make it difficult to ascertain internal cognitive processes, such as those that may be involved in recognizing the structural properties of problems during or prior to incremental search. The integration of gestalt and incremental search approaches is important since real world problems (e.g., those encountered in organizations) are rarely one-step insight problems nor so well defined that problem structure is known a priori. Solving complex real problems usually involves periods of incremental search as well as repeated cycles of frustration, insight and cognitive restructuring.

A second limitation from the perspective of this paper is that the literature has generally emphasized individual rather than group problem solving. Again, the reasons are likely both theoretical and methodological. Group problems are theoretically more complex than individual problems since they are characterized by distributed information and distributed cognition, as well as group social properties (e.g, norms, member status, groupthink, etc.) that may affect behavior and performance by limiting the effectiveness of information sharing. Few suitable 
methods have been designed to incorporate these characteristics of group problem solving, and none address the concerns noted above related to the difficulties of integrating gestalt and incremental search approaches in the context of groups.

To address some of these concerns, this paper presents a view of problem solving based on Heider's balance theory (Cartwright \& Harary, 1956; Heider, 1946, 1958), which conceptualizes problem solving as a progression towards increasing structural balance (or cognitive consistency; Abelson et al., 1968). Balance theory provides a means of representing problems in ways that capture both gestalt restructuring and incremental search properties of problem solving. The group problem solving method developed in this research integrates both cognitive restructuring and incremental search within the same experimental design. The method thereby enables us to manipulate problem structure as an independent variable and examine its effects on group problem solving behavior and performance.

\section{Balance theory}

Balance theory assumes people prefer balanced (consistent, coherent) cognitions (beliefs, attitudes, etc.) over unbalanced (inconsistent, incoherent) cognitions (Heider, 1946, 1958). Heider's theory was based on a gestalt notion of a "good form" (Wertheimer, 1938), whereby different elements of a person's cognition about their immediate situation are conceptually organized in ways that are mutually consistent. Several similar theories of cognitive consistency were proposed following Heider's work including symmetry theory (Newcomb, 1953), congruity theory (Osgood \& Tannenbaum, 1955), cognitive dissonance theory (Festinger, 1957) and others (Abelson et al., 1968), all of which deal with the "structural-dynamic character of human cognition” (Simon \& Holyoak, 2002, p. 283). Recent research has used parallel constraint satisfaction (i.e., connectionist) computer algorithms to model consistency processes in various 
domains (Shultz \& Lepper, 1996; Simon \& Holyoak, 2002; Thagard \& Verbeurgt, 1998).

Research investigating structural properties of social networks have studied balance processes in complex social networks (Doreian \& Hummon, 2003; Doreian, Kapuscinski, Krackhardt, \& Szczypula, 1996; Doreian \& Mrvar, 1996; Hummon \& Doreian, 2003; Hummon \& Fararo, 1995). Balance theory has never been applied directly to problem solving, but Thagard (1989; , 1992) has explained related processes such as scientific reasoning and conceptual change in terms of cognitive coherence.

In Heider's theory cognitions are related to one another positively or negatively based on sentiment relations (e.g. like vs. dislike, approve vs. disapprove) or unit relations (e.g. similar vs. dissimilar, familiar vs. unfamiliar). His primary unit of analysis was a socio-cognitive network of three nodes connected by three links, where nodes represent a focal person $(\mathrm{P})$, another person $(\mathrm{O})$, or some other cognitive units $(\mathrm{X})$ and balance is defined from the perspective of the focal person. Balance corresponds to networks in which the product of the signs of the three links is positive. Unbalanced cognitions, in which the product of signs is negative, are associated with psychological tension, creating pressure to adjust and restructure properties of the cognitive field in ways that achieve balance. For example, if $A$ likes $B(+)$, and $B$ likes $C(+)$, but $A$ dislikes $C(-$ ), the $A-B-C$ network is unbalanced, corresponding to a situation of psychological tension for focal person $A$. $A$ may feel obliged to try and like $C$, or begin to dislike $B$, thereby restructuring the network to achieve balance by adjusting the signs on the $A-B$ or $A-C$ links.

Cartwright and Harary (1956) used graph theory to extend balance theory to networks with more than three nodes to investigate properties of social groups-work that led to significant developments in social network analysis. Among other findings, they showed that "a structure is balanced if and only if its set of points (i.e., nodes) can be partitioned into two 
disjoint subsets such that each positive line joins two points of the same subset and each negative line joins points from different subsets" (Harary, 1959, p. 318). This implies that balanced social groups tend to divide into mutually hostile subgroups, or coalitions, where the members of each subgroup like one another but dislike members of the other subgroup. Noting that social groups can have more than two subgroup coalitions, Davis (1967) generalized this finding by proposing the concept of k-balance, corresponding to a network partitioned into k disjoint subsets where nodes within a subset are positively linked and nodes in different subsets are negatively linked.

Since many empirically observed networks are not balanced, Cartwright and Harary (1956) also proposed measures for the degree of structural balance in a network. In graphs with more than three nodes, the definition of balance generalizes from the positive product of signs around a three-line loop to the positive product of signs around a closed loop or "semi-cycle" of any number of lines. Since complex graphs contain multiple semi-cycles, one measure of the degree of balance is "the ratio of the number of positive semi-cycles to the total number of semicycles" (p. 288). This measure is intuitively appealing but difficult to use in practice since the number of semi-cycles increases exponentially in relation to the number of nodes in a graph. A simpler measure used in most empirical applications is the line index of balance (LIB), defined as the minimum number of lines whose reversal of sign creates a balanced graph (Harary, 1959;

Harary, Norman, \& Cartwright, 1965). Unfortunately, this measure is somewhat counterintuitive in that higher LIB values correspond with lower degrees of balance.

\section{A Balance Theory View of Problem Solving}

Balance theory as extended to complex networks by Cartwright and Harary (1956) has several characteristics that make it particularly amenable to the study of problem solving. With respect to group problem solving, it originated as a social psychological theory and has for the 
most part been applied to situations involving groups and social networks. More significantly, balance theory provides a means of representing the structural and dynamic properties of human cognition, based on the well-established view that humans strive towards cognitive consistency. Moreover, balance is achieved through the restructuring of the network corresponding to a person's cognitive representation of a situation. These properties correspond closely to the properties of problem solving discussed above, namely the dynamic formulation of an appropriate problem structure and dynamic search with a structured problem space.

We propose to use balance network graphs to represent a person's individual, or a group's collective, cognitive representation of problem structure. Further, we propose that problem solving corresponds to a progression from an unbalanced initial state towards a balanced state corresponding with the solution. Each change in sign between two nodes in a balance network simultaneously restructures the network and incrementally increases or decreases the total degree of balance. Thus both gestalt restructuring (i.e., insight) and incremental search properties of problem solving can be represented in the same framework. Insight corresponds with sudden increases in balance associated with significant and rapid restructuring of the network. Incremental search corresponds with slower, more modest restructurings that may increase or decrease total balance, depending on whether search results in intermediate states that are closer to or farther away from the solution.

Measures such as LIB can be used to capture incremental search by tracking changes in the degree of balance during the course of the problem solving process. To demonstrate the utility of balance theory to also capture gestalt restructuring we provide two brief illustrations. First we model the "Necker cube" as a balanced network to show how gestalt figure-ground effects can be represented in this framework. (This example is adapted from one by Thagard 
[1989] which used connectionist methods to demonstrate coherence effects.) Figure-ground effects are fundamental to restructuring in problem solving. By definition, restructuring refers to a change in the cognitive field such that what was background now becomes part of the perceived figure. The Necker cube is shown in Figure 1-A and a representation in terms of a balanced signed-graph (s-graph) is shown in Figure 1-B. Nodes refer to the eight corners of the cube, solid lines refer to positive relations between nodes, and dashed lines refer to negative relations between nodes. Figure-ground effects can be observed in Figure 1-A, where it is possible to perceive either face $\mathrm{ABCD}$ or face $\mathrm{EFGH}$ as the front of the cube, but not both simultaneously. Moreover, most observers can restructure their perception of the cube by reversing which face is at the front and which is at the back. These perceptual effects are represented in the s-graph in Figure 1-B. The graph is balanced as indicated by the fact that it can be partitioned into the two disjoint sets of nodes (A, B, C, D) and (E, F, G, H). The sign relations between nodes imply that if node $\mathrm{A}$ is perceived to be at the front of the cube, nodes $\mathrm{B}, \mathrm{C}$, and $\mathrm{D}$ are also perceived to be at the front while nodes E, F, G, and $\mathrm{H}$ are at the back. Alternatively, if A is perceived to be at the back, B, C, and D are also at the back while E, F, G, and H are at the front.

Insert Figure 1 about here

In the next illustration (Figure 2) we model a simple categorization problem, chosen because the experimental design involves a group categorization problem. Moreover, perceptual grouping is a fundamental cognitive process and Heider's original theory dealt indirectly with categorization through the use of similarity relations as a basis for cognitive balance. Consider 
the task of grouping the following items into categories: sand, camels, ducks and penguins. Sand and camels could be grouped together to form a category of items found in deserts. Then the remaining items ducks and penguins can be grouped to form a category of birds. Alternatively, one might group camels, ducks and penguins together to form a category of animals. Then the remaining item sand could form a category of geological items by itself. Although camels can be grouped either with sand or with ducks and penguins, gestalt figure-ground effects make it difficult to perceive of camels in both categories at the same time. When camels are grouped with sand, then the fact that both are items found in deserts is figured while the fact that camels are animals is pushed to the background. The reverse happens when camels are grouped with ducks and penguins as animals; the fact that camels are found in deserts becomes part of the background.

Insert Figure 2 about here

Now consider a balance theoretic representation of this problem. Intuitively we may wish to specify the relationship between any two similar items as a positive one, resulting in the sgraph in Figure 2-A. While this model is objectively correct in that it accounts for all similarity relationships among the four items, it is inconsistent with the figure-ground effects described above and thus fails to represent accurately how people actually perceive the relationships among the items. S-graphs B and C in Figure 2 provide more accurate representations of how people perceptually group the four items, based on different assumptions about the bases for grouping. S-graph B assumes the categories are desert items and birds; s-graph C assumes the categories are animals and geological items. It would be impossible for an observer to know 
which of the s-graphs B or C most accurately represents a person's grouping of the four items without additional information. Fortunately, in some instances the categories may be inferred by other constraints on the problem. For example, if the goal was to form groups containing as many items as possible, one could infer that the categories were animals and geological items.

\section{Method}

\section{Procedure}

An experimental problem designed to be solved by groups of four participants was devised based on the preceding balance theory conceptualization. The problem was a categorization task in which sixteen cards were distributed evenly among the four participants. Each card had two items (i.e., "clip art" pictures) and the task was for participants to trade cards until each possessed a set of four of kind, using one item from each card as the basis for categorization. Participants sat around a 60" diameter table and were separated by a 12 " high Tbarrier that permitted them to see and speak to one another but blocked their view of one another's cards (see Figure 3). Four video cameras (one located directly above the table and three at other points around the room) and three microphones recorded card exchanges and group discussion.

Insert Figure 3 about here

The T-barrier served several purposes. It ensured that no individual possessed complete information needed to solve the problem, thereby reflecting the distributed information characteristic of real group problem solving situations. It therefore also prevented any single participant from solving the problem individually, ensuring that the solution process involved 
distributed cognition and coordination among all four participants. Finally, it forced participants to share information verbally and to verbalize their thoughts and ideas during the problem solving process. Since group communications were recorded, they provide the experimenter with a degree of access to cognitive processes involved in problem solving which are otherwise internalized and inaccessible to observation.

Participants were undergraduates registered in an organizational behavior course and each group of four solved three different problems during the experiment. Participants were told they would receive "up to $3 \%$ " course bonus marks for participating: $0.5 \%$ per problem if they individually obtained a set of four of a kind, and another $0.5 \%$ per problem if every member of their group ended up with a set of four. During later debriefing, all participants were in fact granted the full $3 \%$. Various incentive structures could be used with this experimental design to vary the relative salience of individual vs. group goals. In the present study our focus was on the effects of problem structure on problem solving behavior and performance, so we simply placed equal weight on individual and group goals.

Other procedural constraints in the experiment included the following. First, groups were not told whether or not a full solution was possible. Second, no time limit was imposed on groups, although it was expected that groups would need up to one hour to solve three experimental problems. In practice, most groups completed three problems in less than an hour, though a few groups took as long as two hours. Third, participants were not required to exchange cards on a strict one-for-one basis. However, to prevent any individual from looking at all of the cards at once, participants were told that no one could have more than six cards or less than two cards in their possession at any time. 


\section{Stimulus and experimental conditions}

The items on the sixteen cards were selected to form categories in various ways depending on experimental condition. Three conditions corresponding with three different problem structures were used in the present experiment: (a) pure card sort, (b) detour, (c) detour plus restructuring. Participants were told nothing about these differences in problem structure. A sample of the stimulus for condition (c) is presented in Figure 4. The correct solution corresponds to the four rows in Figure 4, representing the four sets of animals (cards 1, 2, 3, 4), transportation $(5,6,7,8)$, furniture $(9,10,11,12)$, and shapes $(13,14,15,16)$. Other items on cards served as distractions which were not required to form sets. The initial distribution of cards to group members was held constant across all three conditions, such that each participant possessed one item from each correct solution set: $(1,6,11,15),(2,5,9,13),(3,7,10,14)$, and $(4,8,12,16)$.

A "detour" or blind alley is implemented in Figure 4 using the set of hockey items (cards $1,6,11,16)$ to form a false set that, if collected by one participant, prevents the group as a whole from solving the complete problem. Detours have been investigated in many studies including the earliest work by gestalt psychologists on problem solving behavior in apes and other animals (Bavelas, 1973; Köhler, 1927). Psychologically, detours are difficult for problem solvers to deal with because the correct path to the solution requires the person or group to apparently move further away from the solution. In this experiment the group must dismantle the detour set (hockey), apparently moving away from the solution to be able to form other sets and solve the problem. Recognizing that one is in a detour and recognizing the way out of the detour both require a degree of problem restructuring on the part of problem solvers. 
A more explicit form of cognitive restructuring is implemented in this example using one item per category that is significantly different from the other three, forcing participants to define sets abstractly at a super-ordinate level, rather than at the more obvious basic level of categorization (Rosch, 1978). For example, in the first row of Figure 4 an iguana is grouped with three birds to form the super-ordinate set of animals. In the other three sets a helicopter is grouped with cars, a coat rack with chairs, and a triangle with rectangles. Since it is cognitively simpler for participants to classify swans, chickens, etc., as birds than as animals, they tend to initially perceive these items as birds and see the iguana as irrelevant. After being frustrated in their attempts to come up with a set of four birds, eventually a cognitive restructuring is necessary for them to see the birds and iguana together as a set of animals.

Condition (c) in Figure 4 includes all three dimensions of problem structure used in the present experiment: pure sort, detour and restructuring. Therefore, conditions (a) and (b) were simplifications of this example. In the detour condition (b) the detour category (hockey) was included, but the four items in each of the four solution sets were all basic level items (i.e., the iguana was replaced by a fourth bird, etc.) such that restructuring by abstraction to super-ordinate categories was not required to reach the solution. In the pure sort condition (a) the detour category (hockey) was replaced by random distraction items leaving only four possible sets of four of a kind rather than five. Since participants each solved three different problems, three different versions of the stimulus were constructed for each experimental condition, using different items to form categories in each version. Apart from different items and categories, however, the three stimulus versions were otherwise structurally identical for each condition. 
Besides the items used to form the four solution categories and the detour set, the remaining items on cards were irrelevant to the solution and included as distractions. Distraction items always came in pairs such that they functioned as "mini-detours". The distraction pairs in Figure 4 are: justice (cards 2 and 12), tools $(3,5)$, clocks $(4,14)$, medical devices $(7,9)$, telephone items $(8,15)$, and flowers $(10,13)$.

Finally, prior to beginning the actual experiment each group solved two training problems to become familiar with the problem situation. The training problems used a pure card sort problem structure like condition (a), but the four items in each solution set were identical pictures (e.g., four identical swans rather than four different kinds of birds) to make the problem easier to understand and solve. In the first training problem the T-barrier was not used so participants could see one another's cards and understand the basic requirements of the task. The initial card distribution was also modified to be simpler than the actual experiment, such that each participant initially received two items from one of the solution sets. In the second training problem the T-barrier was introduced and the card distribution was identical to that of the actual experimental conditions.

The stimulus design was inspired by the "five squares problem" devised by Alex Bavelas (1973), a group problem solving task that has been used for training in group cooperation in various settings (Steinbeck, Engel, Ybarra, \& Jansson, 2000). In the five squares problem a variety of geometric shapes of different colors are distributed to five participants whose task is to exchange shapes until each is able to form a complete square. The shapes are configured such that squares can be formed in several different ways, only one of which results in the complete solution. Thus, the formation of incorrect squares places the group in a detour situation such that 
the group must back away from what appears to be a partial solution in order to achieve the complete solution. Although a very interesting problem in many ways, the design of the five squares problem had certain limitations from the perspective of the present study. First some of the shapes are difficult to describe verbally so a group's ability to solve the problem could be hindered by communication problems associated with confusion over shape labels. Second and more significantly, the design is rather static making it difficult to manipulate problem structure as an independent variable. The current stimulus design allows us to compare three conditions with different problem structures, including pure card sort and cognitive restructuring, whereas only the detour property is present in the five squares problem. The current method therefore generalizes the five squares problem to enable the experimental manipulation of various dimensions of problem structure.

\section{Measures}

Two measures were used to evaluate group performance: (a) the time required to reach the solution, and (b) the number of card exchanges required to reach the solution. Two additional measures were derived based on balance theory to track group problem solving behavior. The problem structure was represented as a balance network with positive links between card items within the same set and negative links between card items from different sets. The degree of balance was calculated after each card exchange transaction during the problem solving process, using the line index of balance (LIB) defined earlier (Harary, 1959). Thus, participants began in a state of maximum imbalance and LIB $=0$ corresponded to the correct solution and maximum balance. Since there were four participants per group, we used LIB to measure $\mathrm{k}$ balance for $\mathrm{k}=$ 4 (Davis, 1967). 
Since the problem solving process involves a sequence of card exchanges among the four members of the group, a coding scheme was devised to keep track of these transactions and to compute incremental LIB values. Each card was coded as a three-character string consisting of a letter, a number and another letter. Each card is a member of two different categories, a correct solution category and a false (i.e., detour or distraction) category. The first letter in the code refers to a card's correct solution category, the number uniquely identifies the card in relation to other cards in the solution category, and the second letter refers to the card's false category. In Figure 4 the categories of animals, vehicles, furniture and shapes were coded as A, B, C, and D respectively, and the cards in each of these categories were numbered from 1 to 4 . The detour category (hockey) was coded as category E and the other distraction categories were coded as F, $\mathrm{G}$, etc. Items that force a basic category to be restructured to a super-ordinate category, such as the iguana in category A, were coded as A' instead of A. Based on this coding scheme the three problem structure conditions used in the experiment are given in the Appendix.

LIB was tracked in two ways: (a) objective balance corresponding with the experimenter's view of the problem, counting as positive relations only links between items contained in the four correct solution sets, and (b) subjective balance where links between items in the detour set and in the distraction pairs were also counted as positive relations. Objective balance, therefore, provides an indication of the group's objective progress towards the correct solution. Subjective balance provides an indication of how the participants perceived the problem structure in each experimental condition.

We will use a simple example to illustrate the difference between the two LIB measures. Suppose that a person is initially in possession of the cards A1E, B2E, C3E and D3J (i.e. cards 1, 6, 11, and 15 in Figure 4). In the next step, she obtains the card D4E from another member of the 
group. This gives her a complete set of hockey items so finally she gives away card D3J. Two models of this card exchange sequence are presented in Figure 5. The three s-graphs labeled A1, B1 and $\mathrm{C} 1$ model the subjective view of the participant and s-graphs A2, B2 and C2 model the objective situation as seen by the experimenter. We use LIB to measure the amount of balance at each step in the process. Balance for the group as a whole is defined as $\mathrm{k}$-balance for $\mathrm{k}=4$, so balance for each of the four group members individually is $\mathrm{k}$-balance for $\mathrm{k}=1$, since each person is attempting to form exactly one category of four items. The LIB computation for one participant therefore amounts to counting the number of negative lines in the s-graphs in Figure 5. The four individual LIB values would then simply be added together to compute LIB for the group as a whole. Applying this measure to the subjective model we find that LIB $=3$ for graph $\mathrm{A} 1, \mathrm{LIB}=4$ for graph $\mathrm{B} 1$, and $\mathrm{LIB}=0$ for graph $\mathrm{C} 1$. These values reflect the person's perception that she has formed a complete set of hockey items, suggesting that the group as a whole must be on the correct path towards a full solution. To determine the objective state of affairs, we repeat the measure for the s-graphs in the second model. In this case, LIB $=6$ for graph $\mathrm{A} 2, \mathrm{LIB}=9$ for graph $\mathrm{B} 2$, and $\mathrm{LIB}=6$ for graph $\mathrm{C} 2$. From these values we see that objectively the group is no closer to the full solution after two card exchanges than it was initially, because one group member has fallen into the detour.

Insert Figure 5 about here

Note that positive and negative relations between cards are defined based on the gestalt figure-ground perspective discussed in relation to the earlier illustration. Thus, in graph B1 the link between cards D3J and D4E is negative even though both cards have an item from category 
$\mathrm{D}$ (shapes). This is because it is assumed that the participant views the cards in relation to category E (hockey), so D4E is seen as going together with the other hockey cards rather than with card D3J. In practice, this gestalt perspective amounts to a simple rule stating that when a participant can form card sets in more than one way, s/he is assumed to be paying attention to the category containing the largest number of items. In other words, when LIB values can be computed in more than one way the lower value (i.e., higher balance) is chosen. In graph B2 there is a positive relation between cards D3J and D4E since objectively category $\mathrm{E}$ is a detour that does not contribute to the full solution, so category D is now the largest correct set for the participant.

Plots of subjective and objective LIB trajectories over time provide insight into the path taken by groups to reach the solution. As such they provide a qualitative indication of the difference between participants' perception of the problem structure and its objective structure and a means of tracking both restructuring and incremental search during the problem solving process. Based on the computation of subjective and objective LIB we defined two useful quantitative measures of group problem solving behavior. The first is the total "displacement between subjective and objective LIB" over the course of the problem solving process. This is calculated by subtracting subjective LIB from objective LIB after each card exchange transaction, and summing these differences for all card exchanges. This measure provides an indication of the degree to which a group's subjective understanding of problem structure deviates from the objective structure. A second measure is the number of "direction reversals" in subjective LIB values corresponding to the number of times the slope (i.e., first derivative) of the subjective LIB curve changes sign when plotted against time. That is, since subjective LIB increases and decreases over time as groups search the problem space and move closer to or 
further away from the solution, the number of direction reversals provides an indication of the amount of difficulty a group experiences in solving the problem. The number of reversals also provides a rough indication of the number of times the groups must restructure their collective understanding of the problem. These two variables both provide an indication of the amount of behavioral variety exhibited by the group during the problem solving process.

\section{Experimental Hypotheses}

Since the initial distribution of cards was held constant across all experimental conditions, the identical sequence of card exchanges would result in the correct solution regardless of condition. Since the three problem structures increased in complexity from (a) to (c), however, groups should find them increasingly difficult to solve. This should result in more behavioral variety as groups search the problem space struggling to acquire an appropriate understanding of the problem structure. Likewise, increasing problem structure complexity should be associated with declining problem solving performance corresponding with more time and more card exchanges needed to solve the problem. The experiment therefore tests the following hypotheses:

H1: Problem solving behavioral variety varies directly with problem structure complexity.

H2: Problem solving performance varies inversely with problem structure complexity.

\section{Results}

Subjective and objective LIB trajectories for typical cases of the three experimental conditions are plotted in Figures $6-8$. The charts illustrate how the total displacement between subjective and objective LIB increases with the addition of detour and restructuring in conditions (b) and (c). Similarly, the LIB trajectories exhibit an increasing number of direction reversals 
with the addition of detour and restructuring. In Figure 6 (condition a), LIB decreases (i.e., balance increases) monotonically with time, but in Figure 8 (condition c) the LIB trajectory reverses direction several times. Finally, the amount of time required to solve the problems and the number of card exchanges both increase with the addition of the detour and restructuring dimensions of problem structure.

The LIB trajectories provide an indication of the behavioral and psychological processes involved in problem solving. For instance, in Figure 6 it is apparent that the group's initial conception of the problem structure adequately enabled it to reach the solution. In Figure 8, however, the number and distribution of direction reversals in the subjective LIB trajectory indicate greater problem solving difficulty and two instances of apparent cognitive restructuring. The group initially exchanged one card resulting in an increase in subjective balance but no change in objective balance. It then spent almost five minutes in discussion before making several more exchanges (at about the 300 second point) that reduced both subjective and objective LIB (i.e., increased subjective and objective balance). However, after these moves the group appears to have recognized that it had become stuck and made a few futile attempts to escape the detour (during the 350-500 second period). The group then stopped exchanging cards and spent nearly ten minutes in discussion (during the 500-1000 second period). Through this discussion the group was able to formulate an appropriate conception of the problem structure and subsequently reach the solution using several quick card exchanges during the last minute.

The LIB trajectory in Figure 8 suggests both incremental search and cognitive restructuring occurred during the problem solving process. The flatter portions of the graph indicate periods of incremental search, either through the explicit exchange of cards or through discussion in which various potential solution strategies are explored verbally. The two steep 
downward slopes at about the 300 second and 1000 second points indicate that cognitive restructuring has resulted from the preceding periods of discussion. In the case of the first restructuring, the group appears to have come up with a conception of the problem structure that enabled it to move somewhat closer to the objective solution, but this conception turned out to be incomplete, leading the group into a detour situation. The second restructuring produced the objectively correct conception of the problem structure and enabled the group to escape the detour and reach the solution. Thus, the problem was solved through periods of incremental search punctuated by intermittent restructuring events.

Insert Figures $6-8$ about here

Summary statistics for the four quantitative performance and behavioral measures are presented in Table 1. The amount of time required to solve the problem, the number of card exchanges required to solve the problem, the displacement between subjective and objective LIB, and the number of direction reversals in subjective LIB all increase with increasing complexity in the problem structure. One-way ANOVA results for these four dependent variables indicate significant differences across the three experimental conditions.

Insert Table 1 about here

The LIB displacements suggest there was little difference between groups' subjective perceptions of problem structure and the objective structure in condition (a), larger differences in condition (b), and substantial differences in condition (c). This is consistent with the increased 
structural complexity associated with the addition of detour and restructuring properties and also suggests that the introduction of the restructuring dimension in condition (c) substantially increased the likelihood of groups falling into the detour compared with condition (b). The number of balance reversals indicates that groups found it increasingly difficult to formulate an appropriate model of the problem structure as structural complexity increased from condition (a) to (c). Subjective LIB decreased monotonically for most groups in condition (a), but groups in condition (c) reversed direction an average of six times, suggesting several modifications to their conception of problem structure during the problem solving process.

\section{Discussion and Conclusion}

This paper contributes both theoretically and methodologically towards an integrated view of group problem solving. The social psychology and organizational behavior literature on groups provide relatively little insight into the factors affecting group problem solving behavior and performance. The cognitive psychology literature mainly emphasizes problem solving by individuals rather than groups and has long been divided between gestalt approaches that emphasize cognitive restructuring and insight, and information processing approaches that emphasize incremental search. Drawing on Heider's balance theory (Cartwright \& Harary, 1956; Heider, 1946, 1958) we have proposed a new conceptualization of the problem solving process as a progression towards structural balance, thereby providing a means of integrating gestalt restructuring and incremental search within a single conceptual framework. When a problem is represented as a balance s-graph, each change in sign between two nodes during the problem solving process partially restructures the network and also represents an incremental increase or decrease in total balance. Thus insight in problem solving corresponds with significant and rapid 
restructuring of the network while incremental search corresponds with more modest restructurings.

The experimental method developed for this study operationalizes this conceptual approach in a design that permits both cognitive restructuring and incremental search to be examined at the same time in the context of group problem solving. The method also provides a means of directly manipulating problem structure as an independent variable and examining structural effects on problem solving behavior and performance. Although the notion of problem structure has been a central issue in the problem solving literature for a long time, suitable methods for investigating the effects of problem structure have been lacking, particularly in the domain of group problem solving. The results reported in this paper provide preliminary evidence of important structural effects, but also suggest much remains to be learned about the dimensions of problem structure and their effects on behavior and performance.

The methodological and theoretical approaches developed here open up many avenues for future research. Methodologically, the experimental design offers the opportunity to investigate numerous questions of interest. The stimulus design is flexible and can be modified to investigate in more depth the structural dimensions defined in the present study, for example by examining the effects of more than one detour, or of variable levels of category abstraction in problem restructuring. Other dimensions of problem structure can also be incorporated in the stimulus. For example, in a separate experiment we are investigating the effects of introducing redundancy into the problem structure, by including 5 items in each of the four solution categories. Preliminary results indicate that although this manipulation makes it possible to solve the problem in two different ways, increased search space complexity actually makes the problem more difficult to solve, particularly in cases involving restructuring. As previously 
noted, the group versus individual incentive structure was held constant in the present study, but this could easily be manipulated to investigate the effects of different relative weightings on behavior and performance. For example, many group problem solving situations in organizations are characterized by conflicting individual and group incentives, particular when group members come from different departments with different goals.

Theoretically, the structural balance framework is difficult to apply in many real-world problem solving situations, which are not easily represented in terms of balance s-graphs. Further work is needed to develop systematic approaches for modeling diverse problems and for establishing the boundary conditions on the types of problems that can be represented. Furthermore, the modeling approach used in the present study assumed an objective solution to the experimental problem was known, however, a basic property of problems in real organizational or social policy settings is that objective solutions are unknown. Thus, questions arise about the ability to model problems based on an incomplete specification of the problem structure. On the other hand, as a de facto representation of problem structure, a balance s-graph model of the sort used here may provide a means of structuring ambiguous problem situations, thereby facilitating the problem solving process. More fundamentally, the present research raises theoretical questions about the relationship between balance as defined by Heider $(1946 ; 1958)$ and information processing as used by Newell and Simon (1972) and others. Although the balance theoretic approach provides a means of representing aspects of problem search, no theoretical correspondence between balance and information processing has been proposed in the current research. Whether such a correspondence can be found remains an open question. 


\section{Appendix - The Three Experimental Conditions}

\section{Condition (a) - Pure Card Sort}

\begin{tabular}{||l|l||l|l||l|l|l|l||}
\hline Card & Code & Card & Code & Card & Code & Card & Code \\
\hline 1 & A1E & 2 & A2F & 3 & A3G & 4 & A4H \\
\hline 5 & B1G & 6 & B2I & 7 & B3J & 8 & B4K \\
\hline 9 & C1J & 10 & C2L & 11 & C3F & 12 & C4H \\
\hline 13 & D1L & 14 & D2E & 15 & D3I & 16 & D4K \\
\hline
\end{tabular}

\section{Condition (b) - Detour}

\begin{tabular}{|l|l||l|l||l|l||l|l||}
\hline Card & Code & Card & Code & Card & Code & Card & Code \\
\hline \hline 1 & A1E & 2 & A2F & 3 & A3G & 4 & A4H \\
\hline 5 & B1G & 6 & B2E & 7 & B3I & 8 & B4J \\
\hline 9 & C1I & 10 & C2K & 11 & C3E & 12 & C4F \\
\hline 13 & D1K & 14 & D2H & 15 & D3J & 16 & D4E \\
\hline
\end{tabular}

\section{Condition (c) - Detour and Restructuring}

\begin{tabular}{|l|l||l|l||l|l||l|l||}
\hline \hline Card & Code & Card & Code & Card & Code & Card & Code \\
\hline \hline 1 & A'1E & 2 & A2F & 3 & A3G & 4 & A4H \\
\hline 5 & B1G & 6 & B'2E & 7 & B3I & 8 & B4J \\
\hline 9 & C1I & 10 & C2K & 11 & C'3E & 12 & C4F \\
\hline 13 & D1K & 14 & D2H & 15 & D3J & 16 & D'4E \\
\hline
\end{tabular}




\section{References}

Abelson, R.P., Aronson, E., McGuire, T.W., Newcomb, T.M., Rosenberg, M.J., \& Tannenbaum, O.H. (Eds.), 1968. Theories of cognitive consistency. Holt, Rinehart and Winston, New York.

Bavelas, A., 1950. Communication patterns in task-oriented groups. Journal of the Acoustical Society of America 22(6), 723-730.

Bavelas, A., 1973. The five squares problem: an instructional aid in group cooperation. Studies in Personnel Psychology 5(2), 29-38.

Bavelas, A., Hastorf, A.H., Gross, A.E., \& Kite, W.R., 1965. Experiments on the alteration of group-structure. Journal of Experimental Social Psychology 1(1), 55-70.

Cartwright, D., \& Harary, F., 1956. Structural balance: a generalization of Heider's theory. Psychological Review 63, 277-292.

Cohen, S.G., \& Bailey, D.E., 1997. What makes teams work: group effectiveness research from the shop floor to the executive suite. Journal of Management 23(3), 239-290.

Davis, J.A., 1967. Clustering and structural balance in graphs. Human Relations 20, 181-187.

Doreian, P., \& Hummon, N.P., 2003. Some dynamics of social balance processes: bringing Heider back into balance theory. Social Networks 25, 17-49.

Doreian, P., Kapuscinski, R., Krackhardt, D., \& Szczypula, J., 1996. A brief history of balance through time. Journal of Mathematical Sociology 21, 113-131.

Doreian, P., \& Mrvar, A., 1996. A partitioning approach to structural balance. Social Networks $18,149-168$.

Dunbar, K., 1988. Problem solving. In G. Graham (Ed.), A Companion to Cognitive Science (pp. 289-298). Blackwell, London.

Festinger, L., 1957. A theory of cognitive dissonance. Harper \& Row, New York.

Harary, F., 1959. On the measurement of structural balance. Behavioural Science 4, 316-323.

Harary, F., Norman, R.Z., \& Cartwright, D., 1965. Structural models: an introduction to the theory of directed graphs. Wiley, New York.

Heider, F., 1946. Attitudes and cognitive organization. Journal of Psychology 21, 107-112.

Heider, F., 1958. The Psychology of Interpersonal Relations. Wiley, New York. 
Hendrick, H., 1979. Differences in group problem-solving behaviour and effectiveness as a function of abstractness. Journal of Applied Psychology 64 518-525.

Holloman, C., \& Hendrick, H., 1971. Problem Solving in different sized groups. Personnel Psychology 24(3), 489-500.

Hummon, N.P., \& Doreian, P., 2003. Some dynamics of social balance processes: bringing Heider back into balance theory. Social Networks 25, 17-49.

Hummon, N.P., \& Fararo, T.J., 1995. Assessing hierarchy and balance in dynamic network models. Journal of Mathematical Sociology 20, 145-159.

Ilgen, D.R., Hollenbeck, J.R., Johnson, M., \& Jundt, D., 2005. Teams in organizations: from input-process-output models to IMOI models. Annual Review of Psychology 56, 517-543.

Kaplan, C.A., \& Simon, H.A., 1990. In search of insight. Cognitive Psychology 22, 374-419.

Kohler, W., 1969. The Task of Gestalt Psychology. Princeton University Press, Princeton, NJ.

Köhler, W., 1927. The Mentality of Apes. London.

Laughlin, P., Zander, M., Knievel, E., \& Tan, T., 2003. Groups perform better than the best individuals on letters-to-numbers problems: informative equations and effective strategies. Journal of Personality and Social Psychology 85(4), 684-694.

Laughlin, P., Hatch, E.C., Silver, J.S., \& Boh, L., 2006. Groups perform better than the best individuals on letters-to-numbers problems: effects of group size. Journal of Personality and Social Psychology 90(4), 644-651.

Leavitt, H.J., 1951. Some effects of certain communication patterns on group performance. Journal of Abnormal and Social Psychology 46, 38-50.

MacGregor, J.N., Ormerod, T.C., \& Chronicle, E.P., 2001. Information processing and insight: a process model of performance on the nine-dot and related problems. Journal of Experimental Psychology: Learning, Memory, and Cognition 27(1), 176-201.

Metcalfe, J., \& Wiebe, D., 1987. Intuition in insight and non-insight problem solving. Memory and Cognition 15, 238-246.

Newcomb, T.M., 1953. An approach to the study of communicative acts. Psychological Review 60, 393-404.

Newell, A., \& Simon, H.A., 1972. Human Problem Solving. Prentice-Hall Englewood Cliffs, NJ.

Osgood, C.E., \& Tannenbaum, O.H., 1955. The principle of congruity in the prediction of attitude change. Psychological Review 62, 42-55. 
Rosch, E., 1978. Principles of Categorization. In E. Rosch \& B.B. Lloyd (Eds.), Cognition and categorization. Lawrence Erlbaum Associates Publishers, Hillsdale, NJ.

Shultz, T.R., \& Lepper, M.R., 1996. Cognitive dissonance reduction as constraint satisfaction. Psychological Review 103(2), 219-240.

Simon, D., \& Holyoak, K.J., 2002. Structural dynamics of cognition: from consistency theories to constraint satisfaction. Personality and Social Psychology Review 6(6), 283-294.

Steinbeck, R., Engel, C., Ybarra, C., \& Jansson, E., 2000, June 26-July 1. Team building in globally distributed project-based courses: A case study from the Stanford Learning Lab. Paper presented at EdMedia 2000, Montreal, Quebec.

Swinth, R.L., \& Tuggle, F.D., 1971. A complete dyadic process model of four man group problem-solving. Organizational Behavior and Human Performance 6, 517-549.

Thagard, P., 1989. Explanatory coherence. Behavioural and Brain Sciences 12, 435-467.

Thagard, P., 1992. Conceptual Revolutions. Princeton University Press, Princeton, NJ.

Thagard, P., \& Verbeurgt, K., 1998. Coherence as constraint satisfaction. Cognitive Science 22(1-24).

Wertheimer, M., 1938. Laws of organization in perceptual forms. In W. Ellis (Ed.), A Source Book of Gestalt psychology (pp. 71-88). Routledge \& Kegan Paul, London.

Wertheimer, M., 1982/45. Productive Thinking (Enlarged ed.). University of Chicago Press, Chicago. 


\section{Figure 1}

The Necker Cube and its Representation as a Balanced S-graph
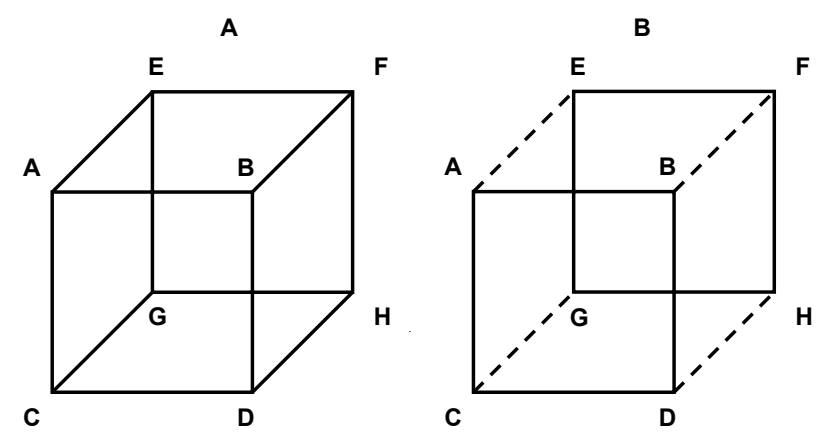

Figure 2

\section{S-graph Representations of a Categorization Problem}

A

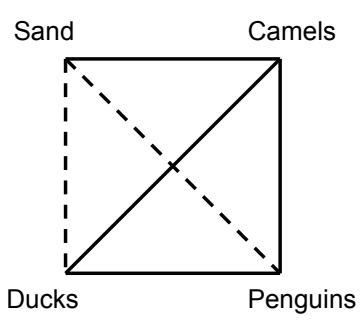

B

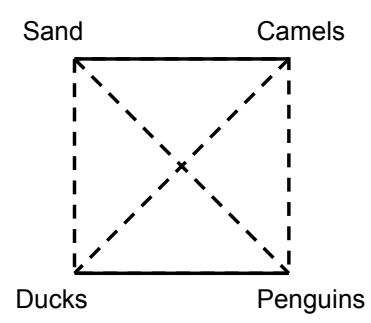

C

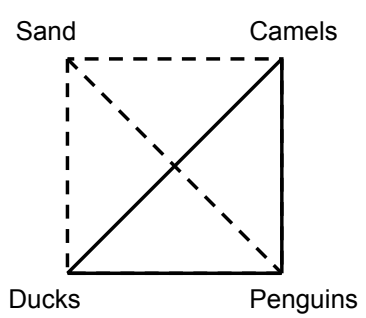


Figure 3

Overhead View of the Experimental Setup

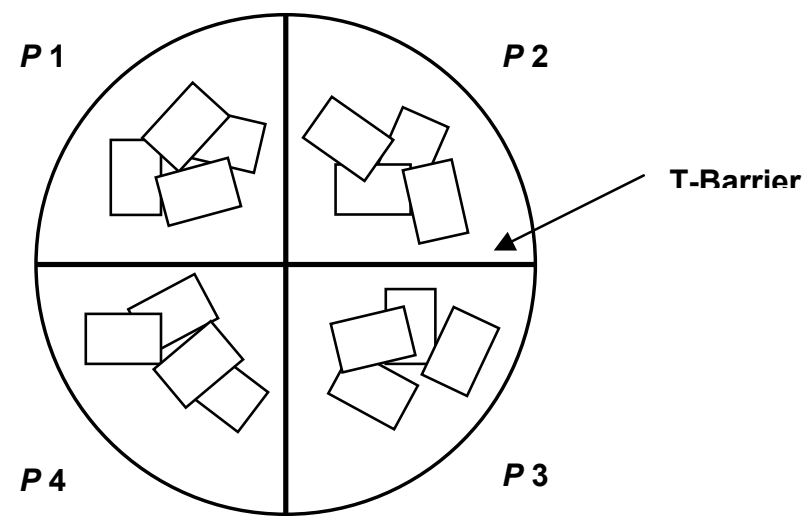

Figure 4

Sample Stimulus for Condition (c)
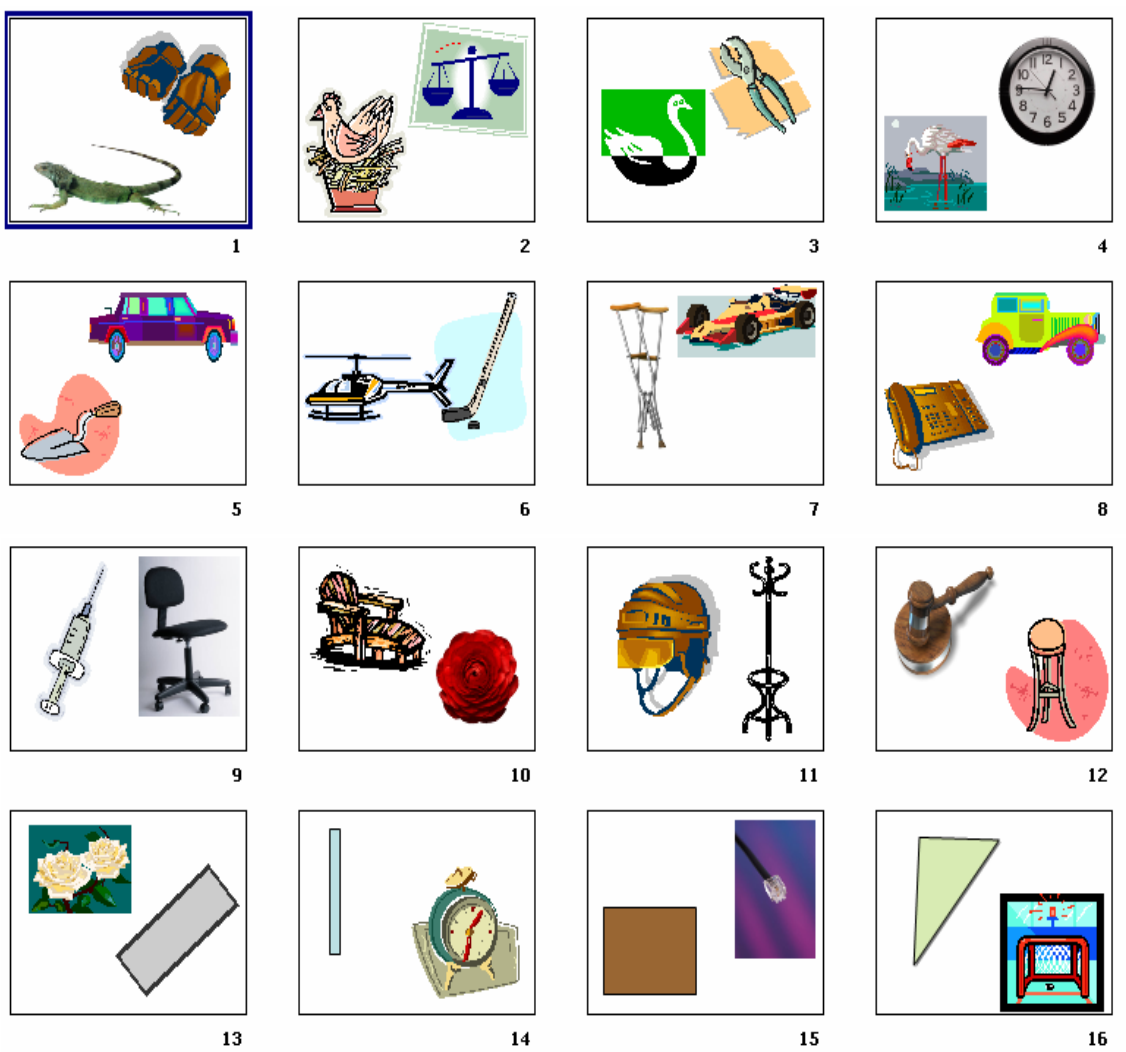
Figure 5

S-graphs Representing Subjective and Objective Progression Towards Category Formation
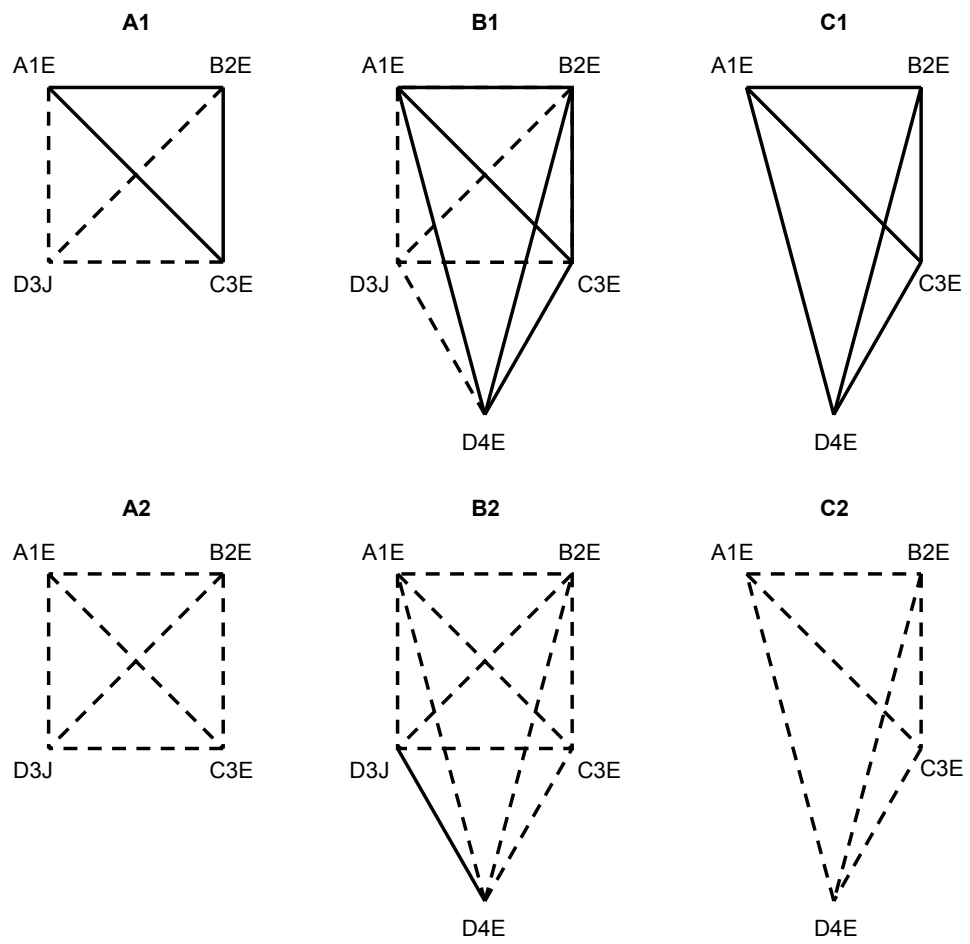


\section{Figure 6}

Subjective and Objective LIB for a Typical Group in Condition (a)

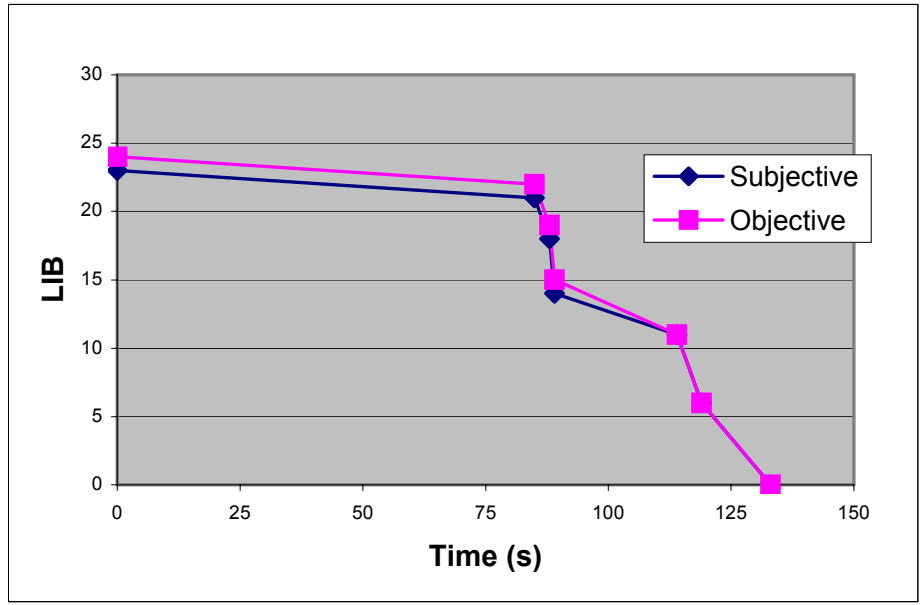

Figure 7

Subjective and Objective LIB for a Typical Group in Condition (b)

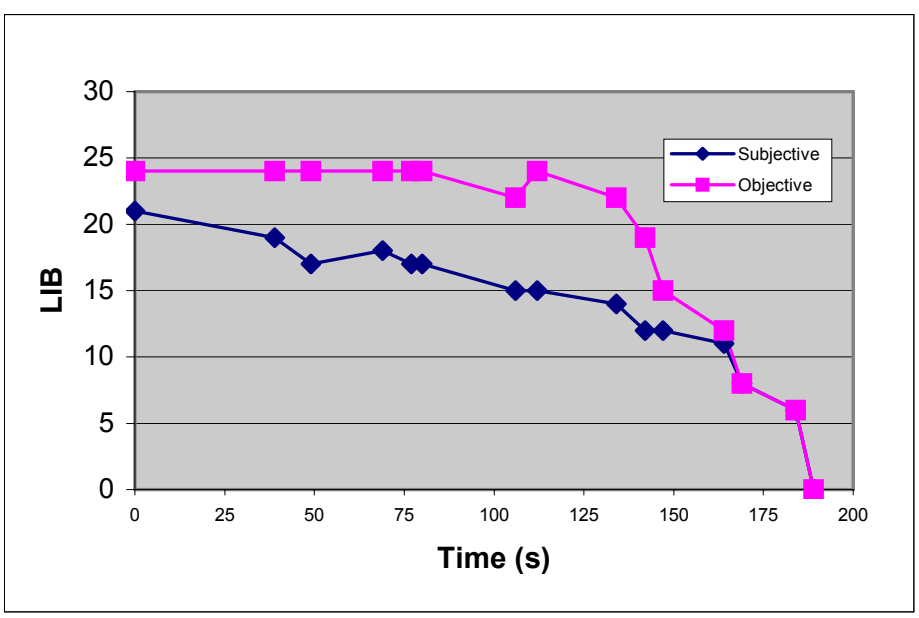




\section{Figure 8}

\section{Subjective and Objective LIB for a Typical Group in Condition (c)}

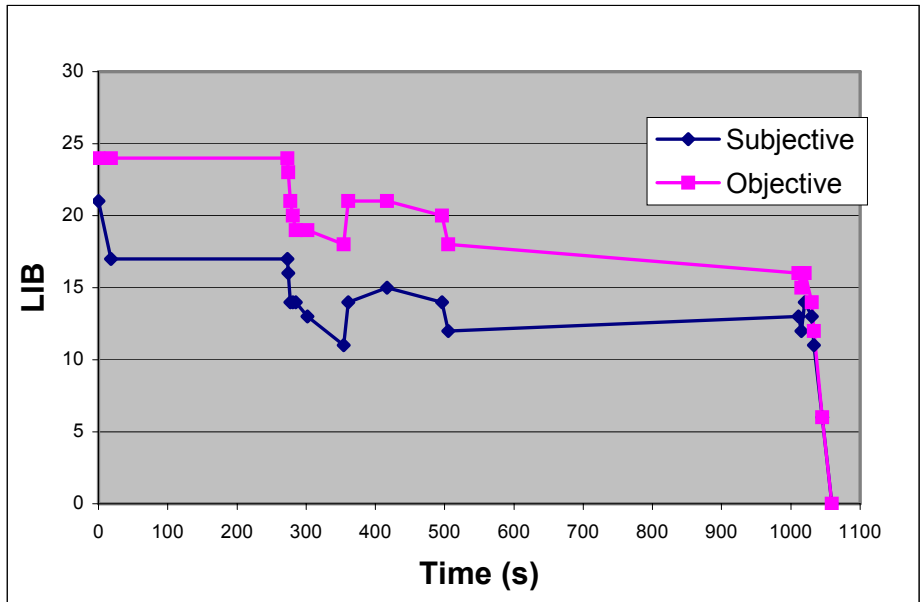

Table 1

Group Problem Solving Performance and Behavioral Variety by Experimental Condition

\begin{tabular}{|c|c|c|c|c|}
\hline $\begin{array}{l}\text { Experimental } \\
\text { Condition }\end{array}$ & $\begin{array}{c}\text { Mean No. of Card } \\
\text { Exchanges to } \\
\text { Reach Solution } \\
\text { (s.d.) }\end{array}$ & $\begin{array}{c}\text { Mean Time } \\
\text { (min:s) to Reach } \\
\text { Solution (s.d.) }\end{array}$ & $\begin{array}{c}\text { Mean Objective } \\
\text { vs. Subjective LIB } \\
\text { Displacement } \\
\text { (s.d.) }\end{array}$ & $\begin{array}{c}\text { Mean No. of } \\
\text { Direction Reversals } \\
\text { in Subjective LIB } \\
\text { Trajectory (s.d.) }\end{array}$ \\
\hline $\begin{array}{l}\text { (a) Pure card sort } \\
(\mathrm{n}=13)\end{array}$ & $12.6(0.96)$ & $02: 50(1: 21)$ & $2.3(1.80)$ & $0.31(0.75)$ \\
\hline $\begin{array}{l}\text { (b) Detour } \\
(\mathrm{n}=15)\end{array}$ & $15.1(6.41)$ & $03: 58(2: 20)$ & $21.3(22.95)$ & $0.57(1.13)$ \\
\hline $\begin{array}{l}\text { (c) Detour \& } \\
\text { Restructuring } \\
(\mathrm{n}=12)\end{array}$ & $25.4(17.78)$ & $12: 53(9: 39)$ & $30.4(29.49)$ & $6.4(7.58)$ \\
\hline F statistic & $5.2 *$ & $11.3 * * *$ & $5.5 * *$ & $13.5 * * *$ \\
\hline
\end{tabular}

Significance levels for one way ANOVA: $* \mathrm{p}<.05 ; * * \mathrm{p}<.01 ; * * * \mathrm{p}<.001$ (between groups $\mathrm{df}=2$ ). 